\title{
Concientizar la importancia del cuidado a los estudiantes de enfermería que presenten astigmatismo, a lograr una mejor educación óptica
}

\author{
Awareness about the importance of visual care for nursing students who present astigmatism, to \\ achieve a better optical education
América Hernández-Bautista ${ }^{a}$, Estefany Hernández-Hernández ${ }^{a}$,Elizabeth Hernández-Lara ${ }^{a}$, Oscar Romero-Salinas ${ }^{a}$, Mayra Vera-Marcos ${ }^{a}$, Dulce M. Mendoza-Melgoza ${ }^{b}$

\begin{abstract}
:
This project focuses on the study of visual problems suffered by nursing students and therefore, has reduced school performance. Through observation, a hypothesis was obtained from the main factor that leads to this problem is the advancement of technology. A survey consisting of 10 items is applied to a specific sample of students, in order to obtain favorable or harmful results that affect their visual health. Thanks to the analysis of the results, it was possible to determine the number of students presenting a condition, highlighting it at a high level with $60.4 \%$ of people with astigmatism problems, thus corroborating these data with a second ophthalmological exam. This condition can be corrected using, contact lenses and surgeries, however, it was decided to provide visual education talks by a specialist trained in this subject, thereby improving their habits.
\end{abstract}

Keywords:

Visual problems, Advancement of technology, Affection, Astigmatism

\section{Resumen:}

Este proyecto se centra en el estudio de los problemas visuales que padecen los alumnos de enfermería y por ello tienen bajo rendimiento escolar. Mediante la observación se obtuvo una hipótesis del principal factor que con lleva a este problema es el avance de la tecnología. Se aplica una encuesta que consta de 10 Ítems a determinada muestra de estudiantes, con el fin de obtener resultados favorables o perjudiciales que afecten a su salud visual. Gracias al análisis de los resultados se pudo determinar la cantidad de alumnos que presentan una afección, resaltándolo en un nivel elevado con un 60.4\% de personas con problemas de astigmatismo corroborando así estos datos con un segundo examen oftalmológico. Esta afección puede corregirse utilizando, lentes de contacto y cirugías, sin embargo, se optó por impartir platicas de educación visual por un especialista capacitado sobre este tema logrando con ello la mejora de sus hábitos.

Palabras Clave:

Problemas visuales, avance de la tecnología, afección, astigmatismo

\footnotetext{
a Universidad Autónoma del Estado de Hidalgo, Escuela Superior de Huejutla, Estudiante de Licenciatura en Enfermería

b Asesor Académico, Universidad Autónoma del Estado de Hidalgo, Escuela Superior de Huejutla, Email: dulce_mendoza@uaeh.edu.mx
} 


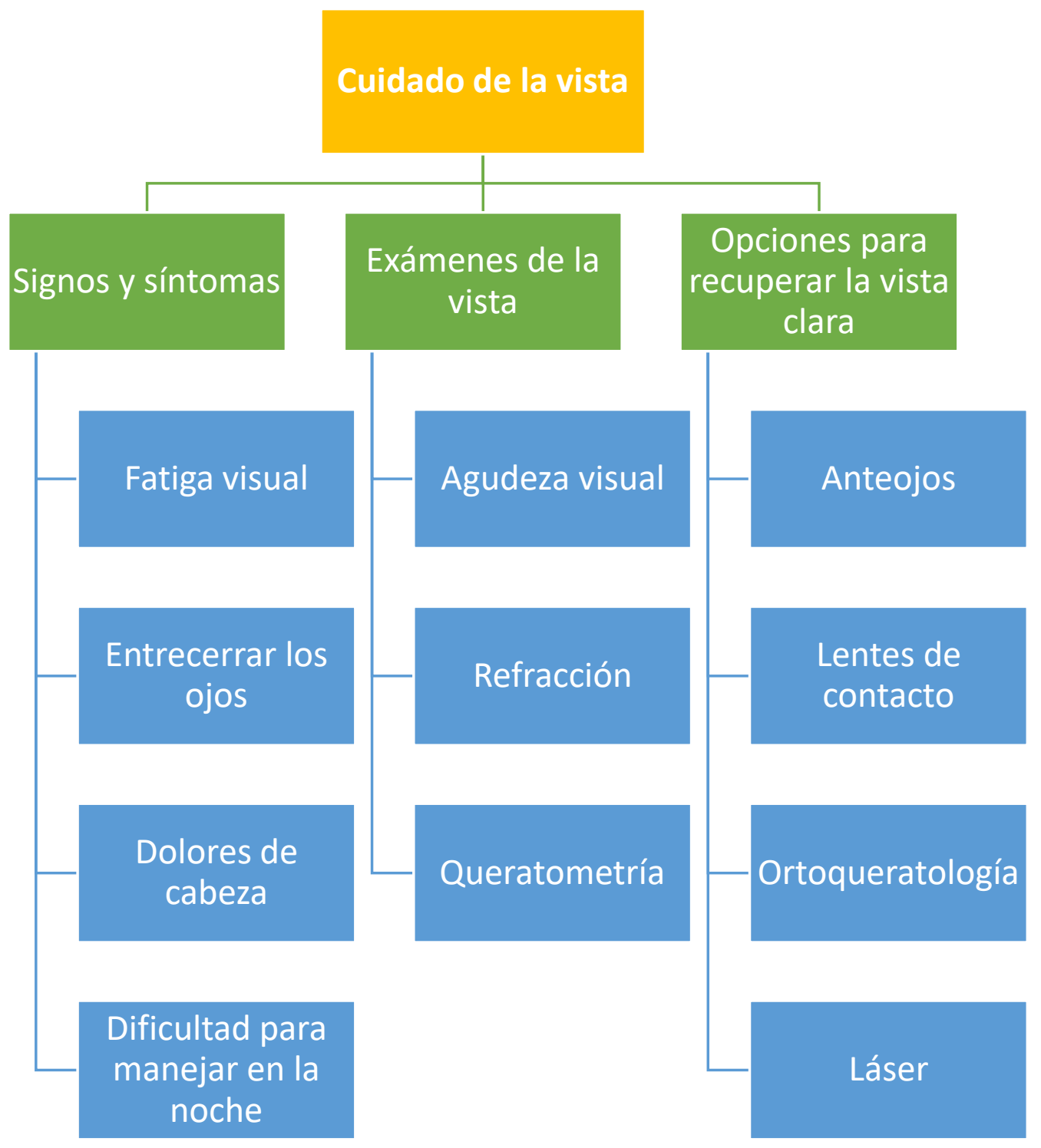

(1) Institute, N. E. (2016). Healthy Vision. Obtenido de National Eye Institute. Research today... Vision tomorrow: https://nei.nih.gov/healthyeyes/spanish/astigmatism_sp

(2) Adhanom, T. (s.f.). Test Astigmatismo. Obtenido de Organización Mundial de la Salud: https://www.who.int/es

(3) Gustavo guglielmontti. (2016). miopía y astigmatismo. españa: openuppray.

(4) Kristine M. Krapp, Jacqueline L. Longe. 28 Jun. 2011. mas astigmatismo . En medicina alternativa (1, 1568 páginas) Universidad de California: Océano. 